\title{
Internet-based behavioural activation to improve depressive symptoms and prevent child abuse in postnatal women (SmartMama): a protocol for a pragmatic randomized controlled trial
}

\author{
Erika Obikane ${ }^{1}$, Toshiaki Baba ${ }^{2 *}$ (1), Tomohiro Shinozaki ${ }^{3}$, Soichiro Obata ${ }^{4}$, Sayuri Nakanishi ${ }^{4}$, Chie Murata $^{5}$, \\ Emiko Ushio ${ }^{5}$, Yukio Suzuki ${ }^{6}$, Norihito Shirakawa ${ }^{7}$, Mari Honda ${ }^{2}$, Natsu Sasaki ${ }^{1}$, Daisuke Nishi ${ }^{1}$, \\ Heather O'Mahen ${ }^{8}$ and Norito Kawakami ${ }^{1}$
}

\begin{abstract}
Background: Child abuse and postnatal depression are two public health problems that often co-occur, with rates of childhood maltreatment highest during the first year of life. Internet-based behavioural activation (iBA) therapy has demonstrated its efficacy for improving postnatal depression. No study has examined whether the iBA program is also effective at preventing child abuse. This study aims to investigate whether iBA improves depressive symptoms among mothers and prevents abusive behaviours towards children in postpartum mothers in a randomized controlled trial, stratifying on depressive mood status. The study also evaluates the implementation aspects of the program, including how users, medical providers, and managers perceive the program in terms of acceptability, appropriateness, feasibility, and harm done.

Methods: The study is a non-blinded, stratified randomized controlled trial. Based on cut-off scores validated on Japanese mothers, participants will be stratified to either a low Edinburgh Postnatal Depression Scale (EPDS) group, (EPDS 0-8 points) or a high EPDS group (EPDS $\geq 9$ points). A total of 390 postnatal women, 20 years or older, who have given birth within 10 weeks and have regular internet-access will be recruited at two hospitals. Participants will be randomly assigned to either treatment, with treatment as usual (TAU) or through intervention groups. The TAU group receives 12 weekly iBA sessions with online assignments and feedback from trained therapists. Coprimary outcomes are maternal depressive symptoms (EPDS) and psychological aggression toward children (Conflict Tactic Scale 1) at the 24-week follow-up survey. Secondary outcomes include maternal depressive symptoms, parental stress, bonding relationship, quality of life, maternal health care use, and paediatric outcomes such as physical development, preventive care attendance, and health care use. The study will also investigate the implementation outcomes of the program.

(Continued on next page)
\end{abstract}

\footnotetext{
* Correspondence: toshiaki-baba@umin.ac.jp

${ }^{2}$ Bureau of International Health Cooperation, National Center for Global Health and Medicine, 1-21-1, Toyama, Shinjuku-ku, Tokyo 162-8655, Japan Full list of author information is available at the end of the article
}

C C The Author(s). 2021 Open Access This article is licensed under a Creative Commons Attribution 4.0 International License, which permits use, sharing, adaptation, distribution and reproduction in any medium or format, as long as you give appropriate credit to the original author(s) and the source, provide a link to the Creative Commons licence, and indicate if changes were made. The images or other third party material in this article are included in the article's Creative Commons licence, unless indicated otherwise in a credit line to the material. If material is not included in the article's Creative Commons licence and your intended use is not permitted by statutory regulation or exceeds the permitted use, you will need to obtain permission directly from the copyright holder. To view a copy of this licence, visit http://creativecommons.org/licenses/by/4.0/. The Creative Commons Public Domain Dedication waiver (http://creativecommons.org/publicdomain/zero/1.0/) applies to the data made available in this article, unless otherwise stated in a credit line to the data. 
(Continued from previous page)

Discussion: The study investigates the effectiveness of the iBA program for maternal depressive symptoms and psychological aggression toward children, as well as implementation outcomes, in a randomized-controlled trial. The iBA may be a potential strategy for improving maternal postnatal depression and preventing child abuse.

Trial registration: The study protocol (issue date: 2019-Mar-01, original version 2019005NI-00) was registered at the UMIN Clinical Trial Registry (UMIN-CTR: ID UMIN 000036864).

Keywords: Behavioural activation, Cognitive behavioural therapy, Postnatal depression, Child abuse, Implementation, Internet-based psychotherapy

\section{Background}

Child abuse is a challenging issue in public health as it affects the physical, psychological, and social health outcomes of individuals for a lifetime [1-9]. From a societal perspective, child abuse imposes a substantial financial burden on societies as it creates high direct and indirect costs in low, middle, and high-income countries [10-12]. Existing research has identified the following characteristics as being associated with a higher risk for child abuse: parental depression, anxiety and anger-hyperactivity, parent stress, poor parent-child interactions, and low social support [13]. However, there is presently no solution for how to prevent child abuse right after birth.

Postnatal depression is another critical problem closely related to child abuse [14, 15]. Depression is the leading cause of disease-related disabilities among women [16, 17]. Previous studies indicated that women with postpartum depression were at higher risk for lower physical and psychological health, substance abuse, and suicidal behaviours [18-22]. Child abuse and postnatal depression often cooccur, and the rate of childhood maltreatment is highest during the first year of life [23, 24]. Children born to depressed mothers were at higher risk for prematurity, delayed physical, motor, and cognitive development [22]. Moreover, postnatal depression among mothers was associated with a significantly increased risk for harsh parenting, physical and psychological abuse [14, 15, 25-27].

Previous evidence has demonstrated that cognitive behavioural therapy (CBT) is effective for improving depressive symptoms among postnatal women [28-33], and emerging evidence has shown that it also reduces maternal stress [34, 35]. Behavioural activation (BA) is a simple but effective psychotherapy that follows the basic CBT principles but does not include cognitive restructuring. The practical principles of BA may be of particular use for postnatal women, who face multiple competing demands on their time and attention. Further, the parsimonious, simple nature of BA supports its straightforward application with a wide range of mental health providers, contributing to its promise as a highly scalable intervention [13]. Moreover, as BA facilitates participants to engage in positive behaviours and works on problem-solving strategies when barriers to implementation of these behaviours are encountered, it may increase positive parent-child interactions, possibly enhancing the bonding relationship between mothers and children and improving child outcomes. In a large study of BA in postnatally depressed mothers who were also randomly assigned to receive either relaxation or a video-feedback parent-child intervention, both conditions resulted in improved cognitive, behavioural and attachment outcomes for the child at 2 years of age [36]. These results suggest that BA may help to support the parent-child relationship underpinning attachment, possibly preventing parental abusive behaviours toward their children.

Internet-based psychotherapy has become an alternative approach to face-to-face psychotherapy or other interventions due to its strengths in accessibility, reach, and cost effectiveness [28, 32, 37-41]. These approaches have gained widespread application during the COVID-19 pandemic. Users can choose their device, time, and place to receive the program depending on their needs. Providers of the program, on the other hand, can deliver the application to the mass target population with fewer human resources and lower cost. A number of studies have now demonstrated that internet-based cognitive behavioural therapy (iCBT) [38, 42], which contains internet-based behavioural activation (iBA) is an effective treatment for prenatal depression, and that iBA itself is an effective treatment for postnatal depression [31, 32].

Internet-based psychotherapy not only reduces practical barriers to access for users but may also reduce psychological barriers. Previous evidence has shown that many women with postnatal depression struggle with stigmatization, and this can be an obstacle for health care providers attempting to connect depressed mothers with medical care $[43,44]$. In qualitative studies of iBA for postnatal depression, mothers who reported struggling with stigma stated that they found internet intervention more acceptable than face-toface intervention [45]. We expect that internet-based interventions including smartphones, which have become such standard communication tools, would 
make it easier for mothers in need to seek care for their mental health. Internet-based psychotherapy, however, has its own challenges. Many studies have described the high attrition rate of internet-based psychotherapy, ranging from 9.7 to $78.9 \%$ of the participants, as a weakness of web-based interventions [40, 46]. Internet-based interventions demonstrated improved retention rates with human support $[28,32$, 41, 47-49]; however, the adequate intensity of support needed to achieve the goal is still unknown. As the implementation of the program involves not only the users but also medical providers and hospital managers, it is essential to understand how the program is perceived in terms of acceptability, appropriateness, feasibility, and potential harm perceived by these stakeholders.

\section{Methods/design Objectives}

The study is a 6-month follow-up stratified randomized controlled trial at multiple medical centres. A web-based behavioural activation program for smartphones with therapist support will be developed for postnatal mothers in Japan, based on a previous study [32]. The aims of the study are as follows:

(1) To investigate the effect of an internet-based behavioural activation program on improving depressive symptoms and preventing abusive behaviours toward their children as co-primary outcomes, as well as parental stress, bonding relationship, healthrelated quality of life (QOL), and child developmental measures as secondary outcomes.

(2) To investigate how the medical and social characteristics of participants influence implementation aspects (acceptability, appropriateness, and feasibility). We have set two co-primary outcomes in this trial, as the effects on both mothers and children are equally important. The hypotheses of the study are as follows:

(H1) The web-based behavioural activation program for smartphones will significantly improve depressive symptoms 24 weeks after the initiation of the program among postnatal mothers with EPDS scores $\geq 9$ points in the intervention group as compared to those in the TAU group; and.

(H2) The smartphone-based behavioural activation program will significantly prevent abusive behaviours toward children at 24 weeks after the initiation of the program among all the postnatal mothers in the intervention group as compared to those in the TAU group.

\section{Study design}

The study is a non-blinded, parallel group, stratified randomized controlled trial. Based on the most recent score of the Edinburgh Postnatal Depression Scale (EPDS) at recruitment, participants will be stratified to either low EPDS group (EPDS 0-8 points) or high EPDS group (EPDS $\geq 9$ points) after completing the baseline survey. The threshold of $8 / 9$ points was determined fit according to the previous validation studies conducted in Japan [50, 51].

The allocation ratio of the intervention group to the control group is set as 1:1. Participants will be asked to answer the online follow-up surveys at 12 and 24 weeks after the initiation of the program. The protocol of the study was registered at UMIN Clinical Trial Registry (UMIN-CTR: ID UMIN 000036864). As of February 2021, we are in the process of recruiting participants. The study protocol follows the Standard Protocol Items for Randomized Trials (SPIRIT) guideline checklists.

\section{Study setting}

The study will be conducted in the department of Obstetrics and Gynecology at two tertiary care hospitals that provide maternal and foetal intensive care in Yokohama, Japan.

\section{Inclusion criteria}

- Age $\geq 20$ years old at recruitment (Age of adulthood is 20 years or older in Japan).

- Postnatal women who had given birth within 10 weeks of recruitment.

- Have regular internet-access via smartphones or other internet devices.

- Currently living with the new-born baby.

- Fluent in Japanese (Able to understand the content and work on the program).

\section{Exclusion criteria}

- Current suicidal intent at recruitment.

- Currently not living with the new-born baby for any reason, including if the baby is still receiving care in the neonatal intensive care unit.

- Currently receiving public livelihood assistance or public assistance for delivery cost.

\section{Procedure}

The flow chart of participants is presented in Fig. 1. Women who have given birth and arrive at the study sites for postnatal visits (usually at 4 weeks after delivery), and fulfilled the eligibility criteria, will be considered as possible candidates for recruitment. Potential candidates will receive handouts with brief information 


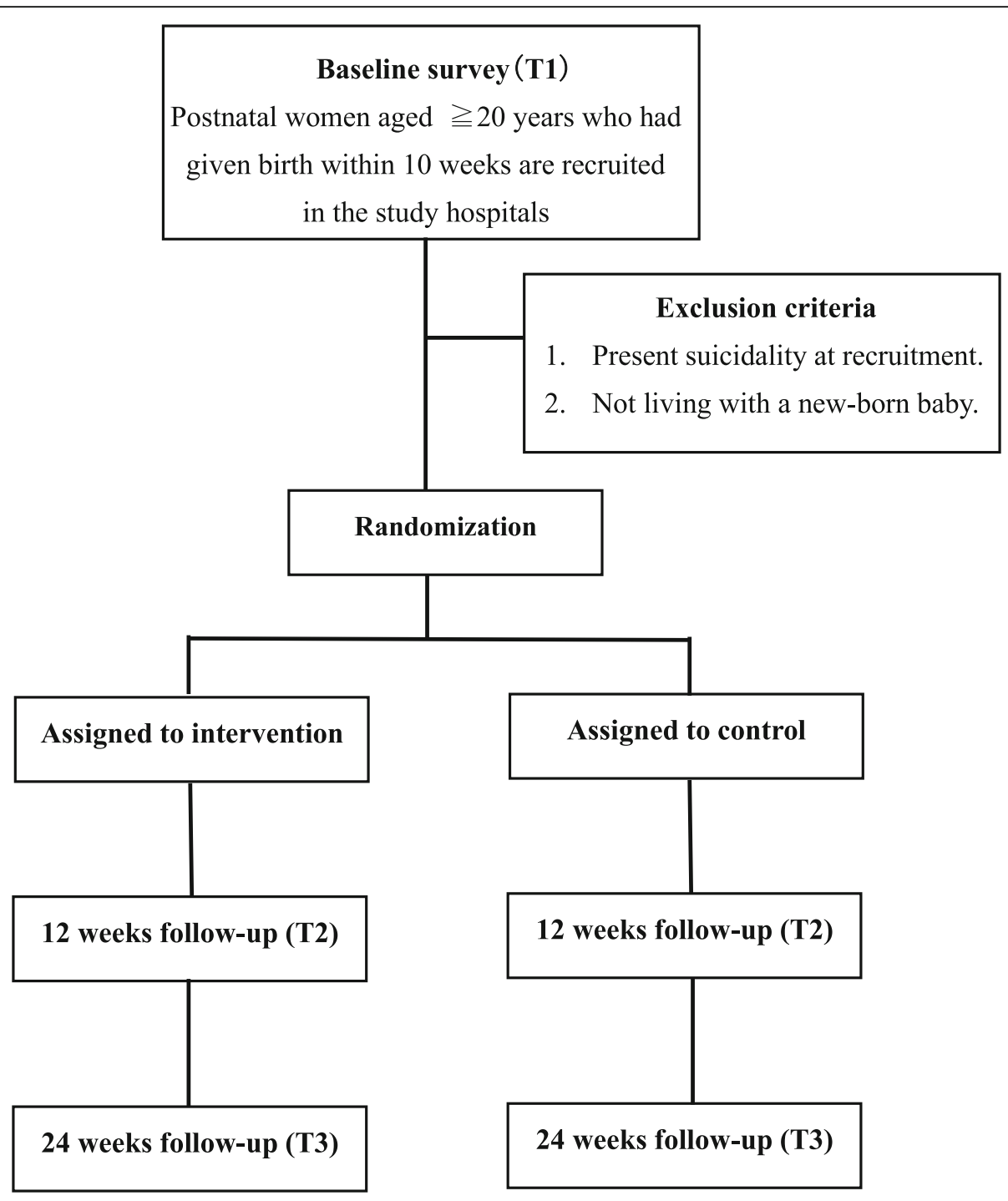

Fig. 1 Flow chart of participants

on our research study from their health care providers (medical doctor, nurse, midwife, or medical assistants). If they are interested in participating in the study, they will be given access to a Quick Respond code. This machine-readable optical barcode can connect to our information site on the internet and register their e-mail addresses for further information. If candidates agree to participate after reading the full information on our study, they will go through the online informed-consent process by confirming that they have read and understood the statements and clicking the "agree" button. Consenting participants will be asked to answer a baseline online survey (T1). Participants will then be allocated to either an intervention group or a treatment as usual (TAU) control group, stratified according to EPDS scores at recruitment (low EPDS: 0-8 points, high EPDS $\geq 9$ points), and hospital codes. To ensure allocation concealment, research assistants will register group codes into the program using the block randomization sheet created by a biostatistician.

This study also conducts a short survey on the implementation of the program with medical providers and hospital managers of the study during the recruitment period. The providers and hospital managers will be asked to browse the content of the program with their own smartphones and answer the short survey consisting of implementation and usability scale outcomes.

\section{Intervention program}

The intervention program (SmartMama) is developed based on the UK behavioural activation program for postnatal depression (Client manual: Self-help booklet for postnatal depression by Heather O'Mahen [52]), adjusted for Japanese postpartum mothers who use 
smartphones [30]. The program consists of 12 weekly online sessions (each session requires $30 \mathrm{~min}$ ) with online assignments and feedback from trained therapists during the course.

The contents of the program are shown in Table 1. In Sessions 1 and 2, participants learn about basic concepts of behavioural activation-that mood, feeling, and behaviours are closely related. They practice how to track their mood, feelings, and actions using a mood diary. In Sessions 3 and 4, participants learn about Triggers-Reactions-Avoidance Patterns (TRAPs) model, how the trigger event leads to a certain mood, often causing avoidance behaviours among participants. Participants break down the patterns of the situations in which they felt stuck, apply the TRAP model to explain their cases, and understand that their actions and mood are closely linked. In Sessions 5 and 6, participants learn to develop alternative coping strategies to handle the situations and shift from avoidance patterns to alternative coping. In Sessions 7 and 8, participants learn about strategies for effective communication and to ask for support. In Sessions 9 and 10, participants identify their goals of being good-enough mothers and turn their avoidance behaviours into alternative coping. In Sessions 11 and 12, participants learn about strategies to stay well, recognize their warning signals, and prepare for the future.

\section{Intervention group}

Participants in the intervention group will be asked to work on 12 weekly sessions of the online application- based behavioural activation programs, as described in the previous section. Each week, participants in the intervention group will receive e-mail reminders that a new session has become available. Further, they will be asked to answer short screening questionnaires on their depressive symptoms each week. If the participants present with severe depressive symptoms and suicidal thoughts, alert messages and information on mental health care will appear on the screen, as well as automatically notifying investigators of the information. If participants have questions about the program, they can ask the therapists within the program. They will receive answers within 1-2 days. Participants are allowed to receive treatment as usual, including inpatient or outpatient consultation, medication, or psychotherapy during the study period.

\section{Control (TAU) group}

Participants in the TAU group receive weekly messages and will be asked to answer short screening questionnaires on their depressive symptoms each week. Similar to the intervention group, alert messages and information on mental health care will be shown if the participants present with severe depressive symptoms or suicidal thoughts. They are also allowed to receive treatment as usual, including inpatient and outpatient medical care.

\section{Outcomes}

The schedule of assessments is summarized in Table 2 . The co-primary outcomes are measured in the baseline,

Table 1 The contents of the behavioral activation therapy program

\begin{tabular}{|c|c|c|}
\hline Sessions & Title & Contents \\
\hline$\overline{0}$ & Introduction & Introduction on changes that occur in perinatal periods that affect your activities \\
\hline 1 & Understanding the depression cycle & $\begin{array}{l}\text { Learning about relationship between your mood and behaviours, and exercise to fill out } \\
\text { the mood diary. }\end{array}$ \\
\hline 2 & Self-monitoring, self-care & Learning about self-monitoring skills. \\
\hline 3 & $\begin{array}{l}\text { Identifying the patterns to get stuck in } \\
\text { you depressive mood }\end{array}$ & Understanding the Triggers-Reactions-Avoidance Patterns (TRAPs) model. \\
\hline 4 & Case study & $\begin{array}{l}\text { Case studies on how a trigger event induces feeling (reaction), causing avoidance pattern. Writing } \\
\text { about your own case using a TRAP model. }\end{array}$ \\
\hline 5 & Altenative coping stratigies & Looking for althernative activities as a mother \\
\hline 6 & Case Study & $\begin{array}{l}\text { Case studies on using alternative coping. Analyse your own case to come up with althernative } \\
\text { behaviours. }\end{array}$ \\
\hline 7 & Support and communication & $\begin{array}{l}\text { Understanding your need for support and how communication needs to be changed during } \\
\text { perinatal period. }\end{array}$ \\
\hline 8 & Communication strategies & Learning communication strategies to ask for help and support in a comfortable way. \\
\hline 9 & Being a "good-enough" mom & Identifying your own "mummy goals" and using althernative coping strategies to meet them. \\
\hline 10 & $\begin{array}{l}\text { TRAPs aroug being a good enough } \\
\text { mom }\end{array}$ & Looking at your TRAP case and how to turn into altenative coping behaviours. \\
\hline 11 & Staying well & Looking back over the course and identify the strategies that were effective and helpful. \\
\hline 12 & Planning ahead & Identify your warning signals and plan your strategies to feel better. \\
\hline
\end{tabular}


Table 2 Schedule of enrolment, interventions, and assessments

\begin{tabular}{llll}
\hline & Enrolment & Allocation & Post-allocation \\
\cline { 2 - 4 } TIMEPOINT & TO & 0 & 12 weeks 24 weeks \\
& & follow-up follow-up \\
(T1) & (T2) & (T1) \\
\hline
\end{tabular}

\section{ENROLMENT:}

Eligibility screen

Informed consent

Allocation

$x$

$x$

INTERVENTIONS:

Intervention group

Control group

\section{ASSESSMENTS:}

\section{Co-primary outcomes}

EPDS

CTS-1

\section{Secondary outcomes}

\section{Maternal}

PHQ-9

PSI-SF

MIBS-J

\section{EQ-5D-5L}

Use of inpatient and outpatient medical care in the past 3 months

\section{Paediatric}

Height, body weight

Type of nutrition (breast milk, formula, both, baby food)

Attendance of well-baby visits at 1, 3, 6-7, 9-10 months)

Immunization record (Hib, pneumococcus, DPT-IPV, rotavirus, BCG)

Use of inpatient and outpatient medical care for injuries, foreign body ingestion, burn, and drowning in the 3 months

\section{Implementation}

Implementation outcome scale for Digital Mental Health (IDMH) for users
Aim

$\begin{array}{ll}\text { Maternal depressive symptoms } & \text { X } \\ \text { Psychological aggression toward } & \text { X } \\ \text { children } & \end{array}$

$x$

$x$

$x$

$x$

Depresssive symptoms

Parent stress

Mother-to-infant bonding

Quality of life

Medical cost

Physical development

Nutritional status

Health check-up status

Immunization status

Medical cost for injuries

Acceptability, appropriateness, feasibility, overall satisfaction, and harms

EPDS Edinburgh Postnatal Depression Scale, CTS-1 Conflict Tactics Scale, PHQ-9 Patient Heatlh Questionnaire-9, PSI-SF Parent stress index-short form Japanese version, MIBS-J Mother-to-Infant Bonding Scale Japanese version, EQ-5D-5L EuroQol-5 dimension-5 level

with 12- and 24-week follow-up online surveys. Participants who have not completed follow-up surveys will receive weekly reminders to complete their surveys.

\section{Co-primary outcomes}

The co-primary outcomes of the study are the depressive symptoms at 24 weeks after initiation of the program and aggression toward their children at 24 weeks after the initiation of the program. We have set two co- primary outcomes, as both maternal and child outcomes were considered equally important.

\section{Depressive symptoms}

The depressive symptoms of participants will be assessed with the EPDS score measured in online surveys. The EPDS is a reliable and valid screening tool that is widely used in perinatal medical and healthcare settings in Japan $[50,53]$. Participants are asked to self-report their 
depressive symptoms on a scale of 0 to 3 for 10 items (total score range being $0-30$ ).

\section{Psychological aggression toward children}

The study uses psychological aggression toward children as outcomes of abusive behaviours toward children. Psychological aggression toward children will be measured by the psychological aggression item of the Conflict Tactic Scale I (CTS-1), a Japanese version developed by Kitamura, et al. [54]. The CTS-1 is a commonly used self-report measure for abusive behaviours toward children that has been tested for its validity $[55,56]$. The participants will be asked to answer the seven items on psychological aggression, on a scale of 0 to 6 , with a higher score indicating more severe psychological aggression.

\section{Secondary outcomes \\ Maternal depressive symptoms}

Depressive symptoms of participants will be evaluated by the Patient Health Questionnaire-9 (PHQ-9). The PHQ-9 is a brief and straightforward self-report instrument to screen and measure the severity of depressive symptoms. The PHQ-9 correlates with the Diagnostic and Statistical Manual of Mental Disorders, Fourth Edition (DSM-IV) criteria for the major depressive disorder $[57,58]$.

\section{Parental stress}

Parental stress will be assessed by the Japanese version of the Parental stress index-short form (PSI-SF). The PSI-SF is a self-report screening tool that consists of 19 questions -9 questions for child characteristics, and 10 questions for parent characteristics on a 5-point Likert scale. A previous study indicates acceptable reliability and internal validity [59].

\section{Bonding relationship}

Mother-to-infant bonding relationship will be measured by the Mother-to-Infant Bonding Scale Japanese version (MIBS-J). The MIBS-J is a self-report questionnaire that consists of 10 questions to assess a mother's feelings toward her child on a 4-point Likert scale. The MIBS-J is widely used in health care settings in Japan, and has acceptable reliability and validity [60].

\section{Physical assault}

Maltreatment measures including both minor and major physical assault are measured with 9 physical assault items of CTS-1 [54-56], as mentioned in the psychological aggression section. The participants will be asked to answer the nine items on physical assault on a scale of 0 to 6 , with a higher score indicating more severe physical assault.

\section{Quality of life}

The quality of life is measured with the EuroQol-5 dimension-5 level (EQ-5D-5L). The EQ-5D-5L is an instrument consisting of five items with five levels to evaluate the health status of the population. The five dimensions include mobility, self-care, usual activities, pain/discomfort, and anxiety/depression. It is widely used and has acceptable reliability and validity according to previous studies [61, 62].

\section{Healthcare use for mothers}

Healthcare use for mothers will be evaluated by questions on the inpatient and outpatient healthcare use for psychiatric problems faced by the participants (the number of days for outpatient visits and hospitalization), or home visitation by public health nurses in the past 3 months during the $12-24$ week surveys.

\section{Paediatric physical development}

The physical development of children will be assessed by standard deviation scores for body weight and height based on the recent records of children's body weight and height during the 12-week and 24-week follow-up surveys.

\section{Health check-up attendance}

Health check-up attendance will be evaluated by asking questions about whether children have attended wellbaby check-ups at 3, 6, and 9 months (if applicable) during the 12-week and 24-week follow-up surveys.

\section{Immunization status}

Immunization status will be evaluated by asking questions about whether children have completed immunization for Hemophilus influenzae type b, Streptococcus pneumoniae, diphtheria, tetanus, pertussis, and polio (DPT-IPV) during the 12-24 week follow-up surveys.

\section{Healthcare use for children}

The healthcare use of children will be evaluated by asking questions about the inpatient and outpatient healthcare use for injuries, fractures, burns, foreign body ingestions, skin problems, or nutrition problems in the past 3 months. Healthcare questions will be administered during the 12-24 weeks of follow-up surveys.

\section{Implementation outcomes}

Implementation outcomes will be measured by the Implementation Outcome Scale for Digital Mental Health (iOSDMH) and the System Usability Scale (SUS). The iOSDMH has been developed to cover the key concepts of implementation in digital mental health, including acceptability, appropriateness, and feasibility, based on a 
previous systematic review [63]. The iOSDMH evaluates these implementation concepts among different levels of stakeholders involved in the intervention process, including users, providers, and managers. Participants will be asked to answer the questionnaires consisting of 19 items from the iOSDMH for users on acceptability, appropriateness, feasibility, and harms, as well as 10 items on usability at the 12-week follow-up survey. Medical providers will be asked to answer the iOSDMH questionnaires that have been specifically developed for providers' perceptions on appropriateness, feasibility, and harm of the program, as well as SUS items. Hospital managers will be asked to answer the iOSDMH questionnaires for managers that have been developed to evaluate the perceptions of managers, as well as SUS items.

\section{Sample size calculation}

As the study has two co-primary outcomes, we calculated each sample size needed to improve the depressive symptoms of mothers and to prevent abusive behaviours toward children based on the effect size from a previous study.

The study reported the effect size of the internetbased behavioural activation for postnatal depression as $\mathrm{d}=0.55$ [32]. With a significance level of 0.05 , the statistical power of $80 \%$, and a follow-up rate of $85 \%$, the sample size needed to detect efficacy for postnatal depression in the high EPDS group is 128 participants (64 participants for each arm). (Sample size calculation was carried out using the $G^{*}$ Power 3.1.9.2.)

No previous study examined the effect of iBA for preventing child abuse, so we used the effect size $d=0.56$ of cognitive behavioural therapy for anger from a randomized controlled trial [64]. We estimated the effect size of the iBA for psychological aggression as $d=0.50$ for the high EPDS group based on the previous study [64], and $\mathrm{d}=0.40$ for the low EPDS group. With a significance level of 0.05 , the statistical power of $80 \%$, and a followup rate of $80 \%$, the required sample size is 150 participants for the high EPDS group (75 participants per arm) and 240 participants for the low EPDS group (120 participants per arm).

Finally, we expected the sample size needed to detect the change in both depressive symptoms and abusive behaviours would be 150 participants for the high EPDS group and 240 participants for the lower EPDS group.

\section{Randomization}

We apply a stratified permuted block randomization for this study. Participants are classified into four strata based on the hospital codes (two hospitals) and the EPDS groups (lower EPDS: 0-8 points, the higher EPDS: $\geq 9$ points). The biostatistician will create a stratified block random table, and the research assistant will allocate participants into either the intervention or TAU group according to the Table. A list of participants allocated to the intervention and TAU groups will be password-locked in the computer and will be blinded to the researchers.

\section{Statistical methods Clinical efficacy}

For primary outcomes, an analysis of covariance (ANCOVA) will be conducted to compare the mean differences of co-primary outcomes at 24 weeks follow-up between the intervention and TAU groups after adjusting baseline scores. The study sets two co-primary outcomes, including the EPDS scores at 24 weeks after the initiation of the program and psychological aggression toward children measured by the CTS- 1 scores at the 24 weeks follow-up survey. The order of statistical testing is as follows: (1) the EPDS scores at 24 weeks followup; (2) CTS-1 scores at 24 weeks follow-up. If we find statistical significance for the EPDS scores at the predefined 0.05 level, then we will conduct statistical testing for the CTS- 1 scores. If we do not find statistical significance for the EPDS scores, we will conduct the ANCOVA for the CTS-1 score as a complementary analysis. Given the fixed-sequence testing procedure, the study will not adjust for the multiplicity of each statistical test.

For secondary outcomes, the ANCOVA will be conducted to compare secondary outcome measures (Table 2) between the TAU and intervention groups after adjusting baseline scores. Statistical analyses will be performed using the SAS version 9.4 (SAS Institute, Cary, North Carolina, USA).

\section{Subgroup analysis}

For primary outcomes, we will conduct a subgroup analysis using initial EPDS, socio-economic factors, adverse childhood experiences, and social support.

\section{Sensitivity analysis}

We will use a complete analysis based upon the assumption missing completely at random. We will conduct a sensitivity analysis using an inverse probability weighted generalized estimating equation to estimate the dropout mechanism. As previous study has indicated that socioeconomic status such as education and household income influenced the attrition rate [30] and we supposed that initial depressive symptoms would possibly influence adherence to the program, this study will use baseline scores of the EPDS, and socio-economic status (highest education, income) as weighting factors. 


\section{Data confidentiality}

Information on individuals acquired through this study will be anonymized. The correspondence table of program ID and individual information will be created and stored in the Department of Mental Health, Graduate School, University of Tokyo. The research data collected in online surveys will be temporarily stored in the Amazon Elastic Compute Cloud system (Amazon Web Services, Inc.). The data will be moved to a passwordlocked PC in the Department of Mental Health, Graduate School, University of Tokyo. Only principal investigators will have access to the final data sets.

\section{Data monitoring}

This study does not have an external data monitoring board; however, we will have regular meetings to discuss our current status of recruitment and data collection with external medical providers and researchers.

\section{Discussion}

The strength of the study is that it evaluates the effectiveness of the iBA program for maternal depressive symptoms and psychological aggression toward children in a randomized-controlled trial. The study also intends to examine the implementation outcomes of the iBA program, which is essential in delivering the program to the population in need. The limitation of the study is that all the outcomes are measured by the self-report scales. Although all the postnatal mothers are recruited in the postnatal check-up at one month after delivery, the time that they complete the baseline online survey and finish enrolment may differ. This may influence the baseline EPDS scores. Moreover, the participants are to be recruited in relatively large hospitals, and the finding of the study may not be generalizable to the postnatal women having deliveries at smaller hospitals and clinics.

The results of the study may be relevant and beneficial to the health care needs of postnatal women and their children, as well as in providing future directions to health policy for postnatal care and child abuse prevention.

\section{Abbreviations}

BA: behavioural activation; CBT: cognitive behavioural therapy; iBA: internetbased behavioural activation; iCBT: internet-based cognitive behavioural therapy; EPDS: Edinburgh Postnatal Depression Scale; TAU: treatment as usual; QOL: quality of life; TRAPs: Triggers-Reactions-Avoidance Patterns; CTS1: Conflict Tactics Scale 1; PHQ-9: Patient Health Questionnaire-9; PSISF: Parental stress index-short form; MIBS-J: Mother-to-Infant Bonding Scale Japanese version; EQ-5D-5L: EuroQol-5 dimension-5 level; DPT-IPV: diphtheria, tetanus, pertussis, and polio; iOSDMH: Implementation outcome scale for Digital Mental Health; SUS: System Usability Scale; ANOVA: analysis of covariance

Acknowledgments

Not applicable.

\section{Authors' contributions}

EO, TB, TS, HO, and NK conceptualized and designed the study; SO, SN, CM, EU, YS, NS1(Norihito Shirakawa), and MH participated in all phases of the study; NS2(Natsu Sasaki) and DN carried out the implementation assessment of the study protocol; $\mathrm{HO}$ conceptualized and reviewed the manuscript. EO, $\mathrm{TB}$, and NK wrote the manuscript. All authors reviewed, revised the manuscript, and approved the final version to be published.

\section{Funding}

Development and management of iBA smartphone programs, and recruitment cost were founded by the Grant-in-Aid for Young Scientists (B) 2017 (Grant number: 17 K15848) from Japan Society for the Promotion of Science. Recruitment and trial equipment were partially funded by Seseragi Foundation, Japan. Maintenance cost of internet server, publication fees and equipment were also supported by internal funding of National Center for Global Health and Medicine, Japan. The funding sources had no role in the study design and will not have any role during recruitment, data collection, analyses, or submission of the results.

Availability of data and materials

The manuscript consists of a trial protocol. Data sharing is not applicable; no datasets were generated or analysed in the preparation of this protocol.

\section{Declarations}

\section{Ethics approval and consent to participate}

The protocol of the study is fully reviewed and approved by the Research Ethics Review Board of Graduate School of Medicine, the University of Tokyo (with reference number: 2019005NI). All the participants are asked to provide online informed consent. The method of consent was approved by the ethics review committee.

\section{Consent for publication}

Not applicable.

\section{Competing interests}

The authors declare that they have no competing interests.

\section{Author details}

'Department of Mental Health, Graduate School of Medicine, The University of Tokyo, Tokyo, Japan. ${ }^{2}$ Bureau of International Health Cooperation, National Center for Global Health and Medicine, 1-21-1, Toyama, Shinjuku-ku, Tokyo 162-8655, Japan. ${ }^{3}$ Department of Information and Computer Technology, Faculty of Engineering, Tokyo University of Science, Tokyo, Japan. ${ }^{4}$ Perinatal Center for Maternity and Neonates, Yokohama City University Medical Center, Yokohama, Japan. ${ }^{5}$ Department of Obstetrics and Gynecology, Yokohama Municipal Citizen's Hospital, Yokohama, Japan. ${ }^{6}$ Department of Obstetrics and Gynecology, Yokohama City University Graduate School of Medicine, Yokohama, Japan. ${ }^{7}$ Yokohama City Mental Health and Welfare Centre, Yokohama, Japan. ${ }^{8}$ Mood Disorders Centre, University of Exeter, Exeter, UK.

Received: 7 February 2021 Accepted: 30 March 2021

Published online: 20 April 2021

\section{References}

1. Anda RF, Croft JB, Felitti VJ, Nordenberg D, Giles WH, Williamson DF, et al. Adverse childhood experiences and smoking during adolescence and adulthood. JAMA. 1999;282(17):1652-8. https:/doi.org/10.1001/jama.282.17.1652.

2. Bruffaerts R, Demyttenaere K, Borges G, Haro JM, Chiu WT, Hwang I, et al. Childhood adversities as risk factors for onset and persistence of suicidal behaviour. British J Psychiatr. 2010;197(1):20-7. https://doi.org/10.1192/bjp. bp.109.074716.

3. Dube SR. Continuing conversations about adverse childhood experiences (ACEs) screening: a public health perspective. Child Abuse Negl. 2018;85: 180-4. https://doi.org/10.1016/j.chiabu.2018.03.007.

4. Dube SR, Anda RF, Felitti VJ, Chapman DP, Williamson DF, Giles WH. Childhood abuse, household dysfunction, and the risk of attempted suicide throughout the life span: findings from the adverse childhood experiences study. JAMA. 2001;286(24):3089-96. https://doi.org/10.1001/jama.286.24.3089. 
5. Felliti VJ, Anda RF, Nordenberg D, Williamson DF, Spitz AM, Edwards V, et al. Relationship of childhood abuse and household dysfunction to many of the leading causes of death in adults. The adverse childhood experiences (ACE) study. Am J Prev Med. 1998;14(4):245-58. https:/doi.org/10.1016/50749-3797(98)00017-8.

6. Gilbert R, Widom CS, Browne K, Fergusson D, Webb E, Janson S. Burden and consequences of child maltreatment in high-income countries. Lancet. 2009;373(9657):68-81. https://doi.org/10.1016/S0140-6736(08)61706-7.

7. Hughes K, Bellis MA, Hardcastle KA, Sethi D, Butchart A, Mikton C, et al. The effect of multiple adverse childhood experiences on health: a systematic review and meta-analysis. Lancet Public Health. 2017;2(8):e356-66. https:// doi.org/10.1016/S2468-2667(17)30118-4.

8. Obikane E, Shinozaki T, Takagi D, Kawakami N. Impact of childhood abuse on suicide-related behavior: analysis using marginal structural models. J Affect Disord. 2018;234:224-30. https://doi.org/10.1016/j.jad.2018.02.034.

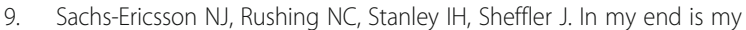
beginning: developmental trajectories of adverse childhood experiences to late-life suicide. Aging Ment Health. 2016;20(2):139-65. https://doi.org/10.1 080/13607863.2015.1063107.

10. Fang X, Brown DS, Florence CS, Mercy JA. The economic burden of child maltreatment in the United States and implications for prevention. Child Abuse Negl. 2012;36(2):156-65. https://doi.org/10.1016/j.chiabu.2011.10.006.

11. Fang X, Fry DA, Brown DS, Mercy JA, Dunne MP, Butchart AR, et al. The burden of child maltreatment in the East Asia and Pacific region. Child Abuse Negl. 2015;42:146-62. https://doi.org/10.1016/j.chiabu.2015.02.012.

12. Wada I, Igarashi A. The social costs of child abuse in Japan. Child Youth Serv Rev. 2014;46:72-7. https://doi.org/10.1016/j.childyouth.2014.08.002.

13. Stith SM, Liu T, Davies LC, Boykin EL, Alder MC, Harris JM, et al. Risk factors in child maltreatment: a meta-analytic review of the literature. Aggress Violent Behav. 2009;14(1):13-29. https://doi.org/10.1016/j.avb.2006.03.006

14. Chaffin M, Kelleher $\mathrm{K}$, Hollenberg J. Onset of physical abuse and neglect: psychiatric, substance abuse, and social risk factors from prospective community data. Child Abuse Negl. 1996;20(3):191-203. https://doi.org/10.1 016/S0145-2134(95)00144-1.

15. Chung EK, McCollum KF, Elo IT, Lee HJ, Culhane JF. Maternal depressive symptoms and infant health practices among low-income women. Pediatrics. 2004;113(6):e523-9. https://doi.org/10.1542/peds.113.6.e523.

16. Kessler RC. Epidemiology of women and depression. J Affect Disord. 2003; 74(1):5-13. https://doi.org/10.1016/S0165-0327(02)00426-3.

17. Murray CJL, Lopez AD. Alternative projections of mortality and disability by causes 1990-2020: global burden of disease study. Lancet. 1997;349(9064): 1498-504. https://doi.org/10.1016/S0140-6736(96)07492-2.

18. Brockington I. Suicide and filicide in postpartum psychosis. Arch Women's Mental Health. 2017;20(1):63-9. https://doi.org/10.1007/s00737-016-0675-8.

19. Chapman SL, Wu LT. Postpartum substance use and depressive symptoms: a review. Women Health. 2013;53(5):479-503. https://doi.org/10.1080/0363 0242.2013.804025

20. Milgrom J, Skouteris H, Worotniuk T, Henwood A, Bruce L. The association between ante- and postnatal depressive symptoms and obesity in both mother and child: a systematic review of the literature. Women's Health Issues. 2012;22(3):e319-28. https://doi.org/10.1016/j.whi.2011.12.001.

21. Orsolini L, Valchera A, Vecchiotti $R$, Tomasetti C, lasevoli F, Fornaro M, et al. Suicide during Perinatal Period: Epidemiology, Risk Factors, and Clinical Correlates. Front Psychiatr. 2016:7(138).

22. Slomian J, Honvo G, Emonts P, Reginster JY, Bruyere O. Consequences of maternal postpartum depression: a systematic review of maternal and infant outcomes. Womens Health (Lond). 2019;15:1745506519844044.

23. The Children's Bureau (Administration on Children Ya, Families AfCaFotUSDoHa, Services. H): Maltreatment 2019. In.; 2019.

24. Wu SS, Ma C-X, Carter RL, Ariet M, Feaver EA, Resnick MB, et al. Risk factors for infant maltreatment: a population-based study. Child Abuse Negl. 2004; 28(12):1253-64. https://doi.org/10.1016/j.chiabu.2004.07.005.

25. Li Y, Long Z, Cao D, Cao F. Maternal history of child maltreatment and maternal depression risk in the perinatal period: a longitudinal study. Child Abuse Negl. 2017:63:192-201. https://doi.org/10.1016/j.chiabu.2016.12.001.

26. McLearn KT, Minkovitz CS, Strobino DM, Marks E, Hou W. The timing of maternal depressive symptoms and Mothers' parenting practices with young children: implications for pediatric practice. Pediatrics. 2006;118(1): e174-82. https://doi.org/10.1542/peds.2005-1551.

27. Zuravin SJ. Severity of maternal depression and three types of mother-tochild aggression. Am J Orthopsychiatr. 1989;59(3):377-89. https://doi.org/1 0.1111/j.1939-0025.1989.tb01673.x
28. Milgrom J, Danaher BG: Internet Cognitive Behavioral Therapy for Women With Postnatal Depression: A Randomized Controlled Trial of MumMoodBooster 2016, 18(3):e54.

29. Ngai FW, Wong PW, Leung KY, Chau PH, Chung KF. The effect of telephone-based cognitive-behavioral therapy on postnatal depression: a randomized controlled trial. Psychother Psychosom. 2015;84(5):294-303. https://doi.org/10.1159/000430449.

30. O'Mahen H, Himle JA, Fedock G, Henshaw E, Flynn H. A pilot randomized controlled trial of cognitive behavioral therapy for perinatal depression adapted for women with low incomes. Depress Anxiety. 2013;30(7):679-87. https://doi.org/10.1002/da.22050

31. O'Mahen HA, Richards DA, Woodford J, Wilkinson E, McGinley J, Taylor RS, et al. Netmums: a phase II randomized controlled trial of a guided internet behavioural activation treatment for postpartum depression. Psychol Med. 2014;44(8):1675-89. https://doi.org/10.1017/S0033291713002092.

32. O'Mahen HA, Woodford J, McGinley J, Warren FC, Richards DA, Lynch TR, et al. Internet-based behavioral activation - treatment for postnatal depression (Netmums): a randomized controlled trial. J Affect Disord. 2013; 150(3):814-22. https://doi.org/10.1016/j.jad.2013.03.005.

33. Sockol LE. A systematic review of the efficacy of cognitive behavioral therapy for treating and preventing perinatal depression. J Affect Disord. 2015;177:7-21. https://doi.org/10.1016/j.jad.2015.01.052.

34. Bleker LS, Milgrom J, Sexton-Oates A, Parker D, Roseboom TJ, Gemmill AW, et al. Cognitive behavioral therapy for antenatal depression in a pilot randomized controlled trial and effects on neurobiological, behavioral and cognitive outcomes in offspring 3-7 years postpartum: a perspective article on study findings, limitations and future aims. Front Psychiatry. 2020;11:34. https://doi.org/10.3389/fpsyt.2020.00034.

35. Milgrom J, Holt CJ, Bleker LS, Holt C, Ross J, Ericksen J, et al. Maternal antenatal mood and child development: an exploratory study of treatment effects on child outcomes up to 5 years. J Dev Orig Health Dis. 2018;10(2): 221-31. https://doi.org/10.1017/S2040174418000739.

36. Stein A, Netsi E, Lawrence PJ, Granger C, Kempton C, Craske MG, et al. Mitigating the effect of persistent postnatal depression on child outcomes through an intervention to treat depression and improve parenting: a randomised controlled trial. Lancet Psychiatry. 2018;5(2):134-44. https://doi. org/10.1016/S2215-0366(18)30006-3.

37. Barrera AZ, Wickham RE, Munoz RF. Online prevention of postpartum depression for Spanish- and English-speaking pregnant women: a pilot randomized controlled trial. Internet Interv. 2015;2(3):257-65. https://doi. org/10.1016/j.invent.2015.06.002.

38. Forsell E, Bendix M, Holländare F, Szymanska Von Schultz B, Nasiell J, Blomdahl-Wetterholm M, et al. Internet delivered cognitive behavior therapy for antenatal depression: A randomised controlled trial. J Affect Disord. 2017;221:56-64. https://doi.org/10.1016/j.jad.2017.06.013.

39. Kelman AR, Evare BS, Barrera AZ, Munoz RF, Gilbert P. A proof-of-concept pilot randomized comparative trial of brief internet-based compassionate mind training and cognitive-behavioral therapy for perinatal and intending to become pregnant women. Clin Psychol Psychother. 2018;25(4):608-19. https://doi.org/10.1002/cpp.2185.

40. Nair U, Armfield NR, Chatfield MD, Edirippulige S. The effectiveness of telemedicine interventions to address maternal depression: a systematic review and meta-analysis. J Telemed Telecare. 2018;24(10):639-50. https:// doi.org/10.1177/1357633X18794332.

41. Pugh NE, Hadjistavropoulos HD, Dirkse D. A randomised controlled trial of therapist-assisted, internet-delivered cognitive behavior therapy for women with maternal depression. PLoS One. 2016;11(3):e0149186. https://doi.org/1 0.1371/journal.pone.0149186.

42. Loughnan SA, Sie A, Hobbs MJ, Joubert AE, Smith J, Haskelberg H, et al. A randomized controlled trial of 'MUMentum pregnancy': internet-delivered cognitive behavioral therapy program for antenatal anxiety and depression. J Affect Disord. 2019:243:381-90. https://doi.org/10.1016/j.jad.2018.09.057.

43. Aboujaoude E, Gega L, Parish MB, Hilty DM. Editorial: digital interventions in mental health: current status and future directions. Front Psychiatry. 2020;11: 111. https://doi.org/10.3389/fpsyt.2020.00111.

44. Clement S, Schauman O, Graham T, Maggioni F, Evans-Lacko S, Bezborodovs $\mathrm{N}$, et al. What is the impact of mental health-related stigma on help-seeking? A systematic review of quantitative and qualitative studies. Psychol Med. 2015;45(1):11-27. https://doi.org/10.1017/S0033291714000129.

45. O'Mahen HA, Grieve H, Jones J, MCGinley J, Woodford J, Wilkinson EL. Women's experiences of factors affecting treatment engagement and 
adherence in internet delivered Behavioural activation for postnatal depression. Internet Interv. 2015;2(1):84-90. https//doi.org/10.1016/jinvent.2014.11.003.

46. Christensen H, Griffiths KM, Farrer L. Adherence in internet interventions for anxiety and depression. J Med Internet Res. 2009;11(2):e13. https://doi.org/1 0.2196/jmir.1194.

47. Lau Y, Htun TP, Wong SN, Tam WSW, Klainin-Yobas P. Therapist-supported internetbased cognitive behavior therapy for stress, anxiety, and depressive symptoms among postpartum women: a systematic review and meta-analysis. J Med Internet Res. 2017;19(4):e138. https:/doi.org/10.2196/jmir.6712.

48. Nieminen K, Berg I, Frankenstein K, Viita L, Larsson K, Persson U, et al. Internet-provided cognitive behaviour therapy of posttraumatic stress symptoms following childbirth - a randomized controlled trial. Cogn Behav Ther. 2016:45(4):287-306. https://doi.org/10.1080/16506073.2016.1169626.

49. Sheeber LB, Seeley JR, Feil EG, Davis B, Sorensen E, Kosty DB, et al. Development and pilot evaluation of an internet-facilitated cognitivebehavioral intervention for maternal depression. J Consult Clin Psychol. 2012;80(5):739-49. https://doi.org/10.1037/a0028820.

50. Okano T, Murata M, Masuji F, Tamaki R, Nomura J. Validation and reliability of prospective study of prevalence, incidence, course and nature. Japanese version of the EPDS. Arch Psychiatr Diag Clin Evaluat. 1988;74:525-33.

51. Yamashita H, Yoshida K, Nakano H, Tashiro N. Postnatal depression in Japanese women. Detecting the early onset of postnatal depression by closely monitoring the postpartum mood. J Affect Disord. 2000;58(2):14554. https://doi.org/10.1016/S0165-0327(99)00108-1.

52. O'Mahen H, Hopley B. Client manual: self-help booklet for postnatal depression. http://psychology.exeter.ac.uk/pmh/. Accessed 15 Dec 2020.

53. Cox JL, Holden JM, Sagovsky R. Detection of postnatal depression. Development of the 10-item Edinburgh postnatal depression scale. British J Psychiatry. 1987;150(6):782-6. https://doi.org/10.1192/bjp.150.6.782.

54. Baba KP, Takauma FR, Tada KP, Tanaka TR, Sakanashi KM, Kataoka YP, et al. Factor structure of the conflict tactics scale 1. Int J Commun Based Nurs Midwifery. 2017;5(3):239-47.

55. Caliso JA, Milner JS. Childhood history of abuse and child abuse screening. Child Abuse Negl. 1992;16(5):647-59. https://doi.org/10.1016/0145-2134(92)90103-X.

56. Straus MA, Hamby SL, Finkelhor D, Moore DW, Runyan D. Identification of child maltreatment with the parent-child conflict tactics scales: development and psychometric data for a national sample of American parents. Child Abuse Negl. 1998;22(4):249-70. https://doi.org/10.1016/S014 5-2134(97)00174-9.

57. Doi S, Ito M, Takebayashi Y, Muramatsu K, Horikoshi M. Factorial validity and invariance of the patient health questionnaire (PHQ)-9 among clinical and non-clinical populations. PLoS One. 2018;13(7):e0199235. https://doi.org/1 0.1371/journal.pone.0199235.

58. Suzuki K, Kumei S, Ohhira M, Nozu T, Okumura T. Screening for major depressive disorder with the patient health questionnaire (PHQ-9 and PHQ-2) in an outpatient clinic staffed by primary care physicians in Japan: a case control study. PLoS One. 2015;10(3):e0119147. https:/doi.org/10.1371/journal.pone.0119147.

59. Araki A, Kanematsu Y, Yokozawa S, Arayashiki R, Aizumi I, Fujishima K. A Study for Developing Parenting Stress Form Scale. J Child Health. 2005;11(1):24-33.

60. Yoshida K, Yamashita H, Conroy S, Marks M, Kumar C. A Japanese version of mother-to-infant bonding scale: factor structure, longitudinal changes and links with maternal mood during the early postnatal period in Japanese mothers. Arch Women's Mental Health. 2012;15(5):343-52. https://doi.org/1 0.1007/s00737-012-0291-1.

61. Herdman M, Gudex C, Lloyd A, Janssen M, Kind P, Parkin D, et al.

Development and preliminary testing of the new five-level version of EQ-5D (EQ-5D-5L). Qual Life Res. 2011;20(10):1727-36. https://doi.org/10.1007/s1113 6-011-9903-X.

62. Ikeda S, Shiroiwa T, Igarashi A, Noto S, Fukuda T, Saito S, et al. Developing a Japanese version of the EQ-5D-5L value set. J Natl Inst Public Health. 2015; 64(1):47-55.

63. Weiner BJ, Lewis CC, Stanick C, Powell BJ, Dorsey CN, Clary AS, et al. Psychometric assessment of three newly developed implementation outcome measures. IS. 2017;12(1):108. https://doi.org/10.1186/s13012-017-0635-3.

64. Howie AJ, Malouff JM. Effects of online cognitive treatment for problematic anger: a randomized controlled trial. Cogn Behav Ther. 2014;43(4):310-8. https://doi.org/10.1080/16506073.2014.939103.

\section{Publisher's Note}

Springer Nature remains neutral with regard to jurisdictional claims in published maps and institutional affiliations.

Ready to submit your research? Choose BMC and benefit from:

- fast, convenient online submission

- thorough peer review by experienced researchers in your field

- rapid publication on acceptance

- support for research data, including large and complex data types

- gold Open Access which fosters wider collaboration and increased citations

- maximum visibility for your research: over $100 \mathrm{M}$ website views per year

At $\mathrm{BMC}$, research is always in progress.

Learn more biomedcentral.com/submissions 